\title{
Evolución del concepto de calidad y los modelos de medición de calidad en el servicio (Evolution of the concept of quality and service quality measurement models)
}

\author{
Arlet Abigail Cruz Sánchez • \\ María Dalia Orduña Carlos * \\ José Gerardo Álvarez Hernández *
}

\begin{abstract}
This article starts with the concept of quality and service, to explain the evolution of the concept of quality. It shows how quality in the service has been an issue that has been the subject of several investigations, a literary review of the quality of service models that have been developed in the quest to quantify intangible goods is presented. Finally, we present some empirical research that has addressed the issue of quality in services.
\end{abstract}

Key words: measuring models, quality, quality service, relational capital, service, social capital

JEL: M10, M31

Resumen. Este articulo parte desde el concepto de calidad y servicio, hasta explicar la evolución que ha tenido el concepto de calidad. Se muestra como la calidad en el servicio ha sido un tema que ha sido motivo de diversas investigaciones, se presenta una revisión literaria de los modelos de medición de la calidad en el servicio que se han desarrollado en la búsqueda por cuantificar los bienes intangibles, por último se presentan algunas investigaciones empíricas que han abordado el tema de la calidad en los servicios.

Palabras clave: calidad en el servicio, capital relacional, capital social, modelos de medición, servicio

\footnotetext{
- Tecnológico Nacional de México, ITCV, Cd. Victoria, Tamps., México,. Email: arlet.abi.18@gmail.com

* Tecnológico Nacional de México, ITCV, Cd. Victoria, Tamps., México,. Email: ordu.carlos90@hotmail.com

* Tecnológico Nacional de México, ITCV, Cd. Victoria, Tamps., México,. Email: alvarezge17@gmail.com
} 


\section{Introducción}

En el actual mundo globalizado, también llamado del conocimiento y de la información la declaración "ninguna empresa es una isla" (Hakansson \& Snehota, 1989; Hakansson \& Snehota, 2006) a más de 25 años de haberse planteado cobra mayor relevancia, los negocios electrónicos, las plataformas interactivas, las redes de contactos, las cadenas de suministros, la integración horizontal y vertical de las organizaciones ha generado un ambiente de competitividad y perfeccionamiento de los procesos de producción y de prestación de servicios; está claro que las empresas requieren interactuar con sus clientes de forma más cercana con relaciones de cooperación, cimentados en soluciones y estrategias conjuntas que los coloquen hacia un ganar-ganar permanente (Zailani \& Rajagopal, 2005).

Las primeras investigaciones sobre calidad, centradas en la propia naturaleza de los servicios, intentan proporcionar modelos conceptuales de calidad de servicio, analizando que es y cómo esta puede medirse. Pero, poco a poco, esas investigaciones van evolucionando hacia modelos más complejos en los que el principal objetivo ya no es únicamente la conceptualización y la medición de la calidad de servicio sino también analizar como esta se relaciona con otros conceptos como la satisfacción del cliente y sus intenciones futuras de comportamiento (Setó, 2004). En la Tabla 1 se muestra algunos autores y sus principales definiciones relacionadas con el tema de calidad en el servicio.

\section{Tabla 1. Principales conceptos del tema de calidad en el servicio}

\begin{tabular}{ll}
\hline Autor & Concepto \\
\hline Kotler (1997, p. 656) & $\begin{array}{l}\text { "El servicio es cualquier actividad o beneficio que una parte ofrece a otra; son } \\
\text { esencialmente intangibles y no dan lugar a la propiedad de ninguna cosa. Su } \\
\text { producción puede estar vinculada o no con un producto físico" }\end{array}$ \\
"Eaither (1983, p. 123) & $\begin{array}{l}\text { "el servicio al cliente, es una gama de actividades que en conjunto, originan una } \\
\text { "La calidad consiste en aquellas características de producto que se basan en las }\end{array}$ \\
Juran (1990) & $\begin{array}{l}\text { necesidades del cliente y que por eso brindan satisfacción del producto". } \\
\text { "Calidad es conformidad con los requerimientos. Los requerimientos tienen que } \\
\text { Crostar claramente establecidos para que no haya malentendidos; las mediciones } \\
\text { deben ser tomadas continuamente para determinar conformidad con esos } \\
\text { requerimientos; la no conformidad detectada es una ausencia de calidad" }\end{array}$ \\
"La calidad del servicio es la diferencia entre las percepciones y las expectativas de \\
Pasuraman, Zeithaml \\
los consumidores"
\end{tabular}

Nota1 . Fuente: Elaboración propia 


\section{Evolución del concepto de calidad}

Reeves y Bednar (1994) revisaron el concepto de calidad concluyendo que no existe una definición universal y global del mismo sino básicamente cuatro tipos de definición destacando la calidad como excelencia, como valor, como ajuste a las especificaciones y como respuesta a la expectativa de los clientes.

a) Calidad como excelencia: en este caso se define como "lo mejor" en sentido absoluto. Esta definición es demasiado abstracta y confusa ya que no orienta a la organización hacia donde debe llevar su gestión. Cabría que los responsables de la organización definiesen el concepto de excelencia aun con el riesgo de no ser igual a la concepción que tendrían los clientes.

b) Calidad como valor: en este caso se segmenta el concepto según el tipo de cliente. Calidad es lo mejor para cada tipo de consumidor. Feigenbaum (1951) sostiene que la calidad de un producto no puede ser considerada sin incluir su coste y que, además, la calidad del mismo se juzga según su precio.

c) Calidad como ajuste a las especificaciones: este concepto surge desde la calidad industrial en la que el producto final debe ajustarse a un patrón preestablecido. La calidad significa asegurar que el producto final es tal como se ha determinado sería, esto es, en base a unas especificaciones previas. A partir de este concepto surge el control estadístico de la producción.

d) Calidad como respuesta a las expectativas de los clientes: esta definición surge del auge de los servicios y la medición de su calidad. Bajo esta premisa se centra el concepto de calidad en la percepción que tiene el cliente. La principal aportación es que se reconoce la importancia de los deseos de los consumidores a la hora de determinar los parámetros que determinan la calidad de un producto o servicio. (Santomà \& Costa, 2007)

La calidad es un término que todos los consumidores entienden pero que difícilmente pueden definir, por lo general se asume por calidad cuando un producto, o servicio, cumple con las funciones o desempeño para el cual fue diseñado o creado. Dentro de este contexto, la calidad es un término relativo multidimensional que supone acepciones diferentes, tanto en el 
tiempo, como en función de quien lo utilice, pues implica el necesario juicio de valor individual y colectivo (Colmenares \& Saavedra, 2007).

Los usuarios finales del producto o servicio opinan que la calidad es la habilidad que la organización debe de tener para que sus productos y servicios cumplan con sus necesidades implícitas. Una necesidad implícita es algo que no está escrito pero se espera ver y recibir, es algo que se da por conocido, por ejemplo: atención, respeto y confiabilidad (Cruz, 2004).

En la Tabla 2 se presentan los diferentes enfoques que se han dado al concepto de calidad.

Tabla 2. Conceptos de calidad y sus diferentes enfoques

\begin{tabular}{|c|c|c|c|}
\hline Autores & Enfoque & $\begin{array}{l}\text { Acento } \\
\text { diferencial }\end{array}$ & Desarrollo \\
\hline Platón & Excelencia & $\begin{array}{l}\text { Calidad absoluta } \\
\text { (producto) }\end{array}$ & $\begin{array}{l}\text { Excelencia como superioridad absoluta }<<\mid 0 \\
\text { mejor }>>\text {. Asimilación con el concepto de } \\
<<\mid u j o>>\text {. Analogía con la calidad de diseño. }\end{array}$ \\
\hline $\begin{array}{l}\text { Shewhart } \\
\text { Crosby }\end{array}$ & $\begin{array}{l}\text { Técnico: conformidad } \\
\text { con especificaciones }\end{array}$ & $\begin{array}{l}\text { Calidad } \\
\text { comprobada / } \\
\text { controlada } \\
\text { (procesos) }\end{array}$ & $\begin{array}{l}\text { Establecer especificaciones. Medir la calidad } \\
\text { por la proximidad real a los estándares. } \\
\text { Énfasis en la calidad de conformidad. Cero } \\
\text { defectos. }\end{array}$ \\
\hline $\begin{array}{l}\text { Deming } \\
\text { Taguchi }\end{array}$ & $\begin{array}{l}\text { Estadístico: Pérdidas } \\
\text { mínimas para la } \\
\text { sociedad, rediciendo la } \\
\text { variabilidad y mejorando } \\
\text { estándares }\end{array}$ & $\begin{array}{l}\text { Calidad generada } \\
\text { (producto y } \\
\text { procesos) }\end{array}$ & $\begin{array}{l}\text { La calidad es inseparable de la eficacia } \\
\text { económica. Un grado predecible de } \\
\text { uniformidad y fiabilidad a bajo coste. La } \\
\text { calidad exige disminuir la variabilidad de las } \\
\text { características del producto alrededor de los } \\
\text { estándares y su mejora permanente. } \\
\text { Optimizar la calidad de diseño para mejorar la } \\
\text { calidad de conformidad. }\end{array}$ \\
\hline $\begin{array}{l}\text { Feigenbaum } \\
\text { Juran } \\
\text { Ishikawa }\end{array}$ & Aptitud para el uso & $\begin{array}{l}\text { Calidad } \\
\text { planificada } \\
\text { (sistema) }\end{array}$ & $\begin{array}{l}\text { Traducir las necesidades de los clientes en las } \\
\text { especificaciones. La calidad se mide por } \\
\text { lograr la aptitud deseada por el cliente. } \\
\text { Énfasis tanto en la calidad de diseño como de } \\
\text { conformidad. }\end{array}$ \\
\hline $\begin{array}{l}\text { Parasuraman } \\
\text { Berry } \\
\text { Zeithaml }\end{array}$ & $\begin{array}{l}\text { Satisfacción de las } \\
\text { expectativas del cliente }\end{array}$ & $\begin{array}{l}\text { Calidad } \\
\text { satisfecha } \\
\text { (servicio) }\end{array}$ & $\begin{array}{l}\text { Alcanzar o superar las expectativas de los } \\
\text { clientes. Énfasis en la calidad de servicio. }\end{array}$ \\
\hline $\begin{array}{l}\text { Evans } \\
\text { (Procter \& } \\
\text { Gamble) }\end{array}$ & Calidad total & $\begin{array}{l}\text { Calidad } \\
\text { gestionada } \\
\text { (empresa y su } \\
\text { sistema de valor) }\end{array}$ & $\begin{array}{l}\text { Calidad significa crear valor para los grupos } \\
\text { de interés. Énfasis en la calidad de toda la } \\
\text { cadena y el sistema de valor. }\end{array}$ \\
\hline
\end{tabular}

Nota 2. Fuentes: (Camisón, Cruz, \& González, 2006) 


\section{Modelos de medición de la calidad del servicio}

Las diferentes perspectivas conceptuales de la calidad pueden organizarse en dos categorías, según hablen de calidad objetiva y calidad subjetiva. La calidad objetiva deriva de la comparación entre un estándar y un desempeño, referidos a características de calidad medibles cuantitativamente con métodos ingenieriles 0 tecnológicos. Este concepto describe bien la excelencia, bien la superioridad técnica de los atributos del producto o del proceso, siendo independiente de la persona que realiza la medición 0 adquiere el producto. En cambio, la calidad subjetiva se basa en la percepción y en los juicios de valor de las personas, y es medible cualitativamente estudiando la satisfacción del cliente (Camisón, Cruz, \& González, 2006).

A diferencia de la calidad en los productos, que puede ser medida objetivamente a través de indicadores tales como duración o número de defectos, la calidad en los servicios es algo fugaz que puede ser difícil de medir (Parasuraman et al., 1988). La propia intangibilidad de los servicios origina que éstos sean percibidos en gran medida de una forma subjetiva (Grönroos, 1994). En la Tabla 3 se presenta un resumen cronológico de los modelos de medición más relevantes que se han creado.

Tabla 3. Resumen cronológico de los más relevantes aportes sobre la medición de la calidad del servicio

\begin{tabular}{ccc}
\hline Año & Autores & Aportes \\
\hline 1980 & Oliver & Paradigma Desconfirmatorio \\
1984 & Grönroos & Modelo de la Calidad de Servicio \\
1985,1988 & Parasuraman, Zeithaml y Berry & Modelo SERVQUAL \\
1989 & Elglier y Langeard & Modelo de Servucción \\
1992 & Cronin y Taylor & Modelo SERVPERF \\
1993,1994 & Teas & Modelo de Desempeño Evaluado \\
1994 & Rust y Oliver & Modelo de los Tres Componentes \\
1996 & Dabholkar, Thorpe y Rentz & Escala Multinivel de Calidad del Servicio \\
1997 & Philip y Hazlett & Modelo P-C-P \\
2001 & Brady y Cronin & Modelo Jerárquico Multidimensional \\
2006 & Akbaba & Técnica del Factor Incidente Crítico \\
2014 & Balmori y Flores & Modelo de Evaluación de la Calidad al Servicio \\
& & Interno (MECSI) \\
\hline
\end{tabular}

Nota 3. Fuente: Elaboración propia con base en Colmenares \& Saavedra (2007) 


\section{El modelo servqual (service quality)}

El modelo de la escuela americana de Parasuraman, Zeithaml y Berry se ha denominado SERVQUAL. Es sin lugar a dudas el planteamiento más utilizado por los académicos hasta el momento, dada la proliferación de artículos en el área que usan su escala. Parasuraman, Zeithaml y Berry $(1985,1988)$ partieron del paradigma de la desconfirmación, al igual que Grönroos, para desarrollar un instrumento que permitiera la medición de la calidad de servicio percibida. Luego de algunas investigaciones y evaluaciones, tomando como base el concepto de calidad de servicio percibida, desarrollaron un instrumento que permitiera cuantificar la calidad de servicio y lo llamaron SERVQUAL. Este instrumento les permitió aproximarse a la medición mediante la evaluación por separado de las expectativas y percepciones de un cliente, apoyándose en los comentarios hechos por los consumidores en la investigación (Duque, 2005).

A partir de un grupo extenso de entrevistas identificaron diez determinantes de la calidad del servicio. Pero en los análisis estadísticos que se realizaron para la consecución de SERVQUAL se mostró la correlación existente entre algunas de las diez dimensiones iniciales propuestas por el estudio cualitativo. Como consecuencia de esta correlación se procedió a la consolidación de algunos ítems, quedando cinco: elementos tangibles, fiabilidad, capacidad de respuesta, seguridad y empatía (Lloréns, 1995). En la Tabla 4 se muestran las cinco dimensiones del modelo servqual y su respectiva descripción.

Tabla 4. Dimensiones del modelo servqual

\begin{tabular}{ll}
\hline Dimensión & Descripción \\
\hline Elementos Tangibles & $\begin{array}{l}\text { Apariencia de las instalaciones físicas, equipo, personal y materiales } \\
\text { para comunicaciones }\end{array}$ \\
Confiabilidad & $\begin{array}{l}\text { Capacidad para brindar el servicio prometido en forma precisa y digna } \\
\text { de confianza }\end{array}$ \\
Capacidad de respuesta & $\begin{array}{l}\text { Buena disposición para ayudar a los cliente a proporcionarles un } \\
\text { servicio expedito } \\
\text { Seguridad }\end{array}$ \\
Conocimiento y cortesía de los empleados, así como su capacidad \\
para transmitir seguridad y confianza \\
Cuidado y atención individualizada que la empresa proporciona a sus \\
clientes
\end{tabular}

Nota 4. Fuente: Schiffman \& Kanuk (2010) 
En la Figura 1 se muestra un esquema del modelo de medición de la calidad del servicio servqual.

Figura 1. Esquema del modelo servqual

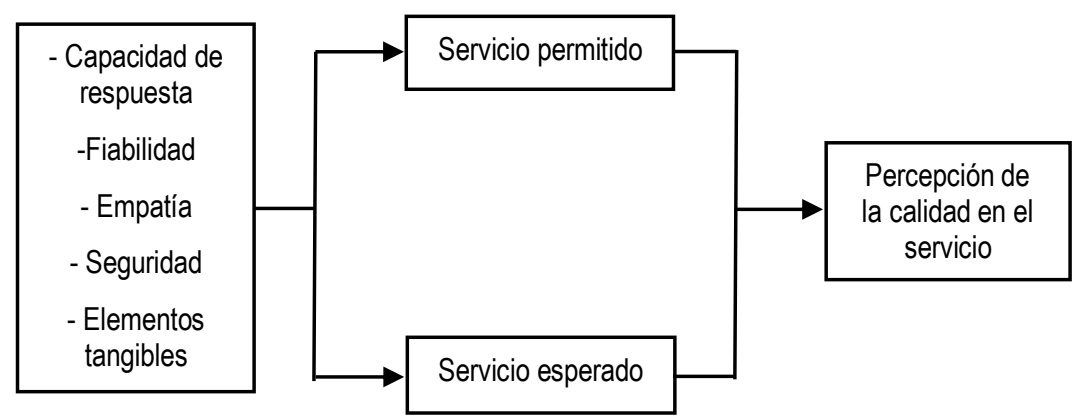

Fuente: (Hernández \& Ulibiarri, 2015), con base en (Zeithaml, Parasuramar y Berry, 1993)

El cuestionario del modelo servqual se divide en tres secciones, la primera mide las expectativas que el cliente tiene sobre un servicio determinado, la segunda mide la percepción del cliente en cuanto a la empresa que brinda el servicio y la tercera permite al cliente calificar el servicio mediante las cinco dimensiones respecto a su perspectiva.

\section{Modelo de las deficiencias}

Otro modelo de medición del servicio es el modelo de la escuela norteamericana de calidad del servicio desarrollado por Parasuraman et al. (1985), también llamado Modelo de las deficiencias o de los Gaps, el cual tiene como finalidad identificar las causas del servicio al cliente. De acuerdo al modelo ya mencionado, las diferencias entre las expectativas del cliente y su percepción del servicio denominado Gap 5, pueden tener su origen en cuatro dimensiones:

Gap 1: Desacuerdo entre las expectativas de los clientes y la percepción que tiene la empresa de esas expectativas.

Gap 2: Desacuerdo de la empresa entre las expectativas que tienen los clientes y las especificaciones de calidad del servicio.

Gap 3: Desacuerdo entre las especificaciones de calidad, diseño del servicio y el servicio ofrecido. 
Gap 4: Desacuerdo entre el servicio ofrecido por la empresa y lo que se comunica a los clientes.

Los Gaps del 1 al 4 nombrados como deficiencias internas colaboran a la aparición del Gap 5 denominado deficiencia percibida por los clientes en la calidad del servicio. Las empresas deberán disminuir los Gaps 1 al 4 y mantenerlas en su nivel más bajo con el objetivo de que no se llegue a presentar el Gap número 5.

\section{Modelo servperf (service performance)}

Cronin y Taylor (1992) establecieron una escala más concisa que SERVQUAL y la llamaron SERVPERF. Esta nueva escala está basada exclusivamente en la valoración de las percepciones, con una evaluación similar a la escala SERVQUAL, la cual tiene en cuenta tanto las expectativas como las percepciones (Duque, 2005). Cuestionaron las bases conceptuales de la escala SERVQUAL y la encontraron confusa con la satisfacción del servicio. Por lo tanto, opinaron que el componente de las expectativas de SERVQUAL debe ser descartado y solo deben ser usadas las percepciones. Propusieron que se llamara a esta escala SERVPERF.

Además de los argumentos teóricos proporcionaron evidencia empírica a través de cuatro industrias (bancos, control de plagas, tintorería y comida rápida) para confirmar la superioridad de su instrumento basado solo en las percepciones sobre la escala SERVQUAL basada en la desconfirmacion. Comenzando como una variante de la escala SERVQUAL y conteniendo solo el componente de percepciones, la escala se compuso de solo 22 items (Jain \& Gupta, 2004 ).

\section{Modelo de la imagen}

Este modelo fue formulado por Grönroos $(1988,1994)$ y relaciona la calidad con la imagen corporativa. El cliente está influido por el resultado del servicio, pero también por la forma en que lo recibe y la imagen corporativa. Todo ello estudia transversalmente las diferencias entre servicio esperado y percepción del servicio. Una buena evaluación de la calidad percibida se obtiene cuando la calidad experimentada cumple con las expectativas del cliente, es decir, lo satisface. 
Según Grönroos (1994, p. 38), la experiencia de calidad es influida por la imagen corporativa/local y a su vez por otros dos componentes distintos: la calidad técnica y la calidad funcional. La calidad técnica se enfoca en un servicio técnicamente correcto y que conduzca a un resultado aceptable. Se preocupa de todo lo concerniente al soporte físico, los medios materiales, la organización interna.

Es lo que Grönroos denomina la dimensión del "qué". Lo que el consumidor recibe. La calidad funcional se encarga de la manera en que el consumidor es tratado en el desarrollo del proceso de producción del servicio. Es la dimensión del "cómo" la manera en que el consumidor recibe el servicio. La forma en que los consumidores perciben la empresa es la imagen corporativa de la empresa. (Duque, 2005)

El Modelo de la Imagen de Gronroos (1982, 1994), que se encarga de relacionar la calidad con la imagen corporativa, ya que dicha imagen puede determinar aquello que se espera del servicio prestado por una organización. El modelo plantea que la calidad total percibida por los clientes es la integración de la calidad técnica' (qué se da), la calidad funcional' (cómo se da) y la imagen corporativa. Por lo tanto, Gronroos piensa que la imagen es un elemento más a tener en cuenta para medir la calidad (Camisón, 1999). En la Figura 2 se muestra un esquema con los componentes del modelo de la imagen.

Figura 2. Esquema del modelo de la imagen

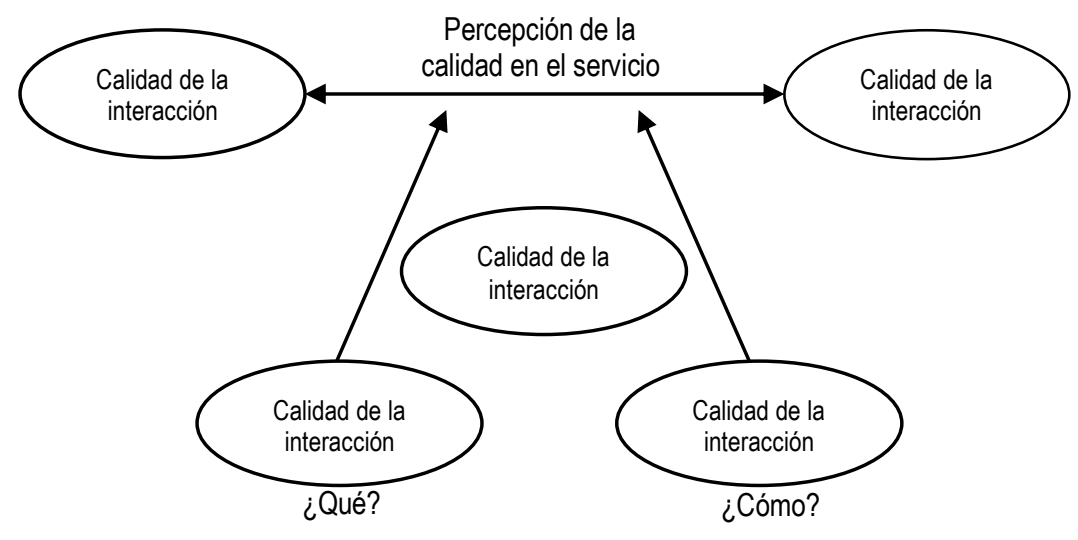

Fuente: Grönrroos (1984) 


\section{Modelo de los tres componentes}

Rust y Oliver (1994) presentaron una conceptualización no probada, pero que fundamenta lo planteado por Grönroos. Su justificación está en las evidencias encontradas por autores como (McDougall y Levesque, 1994) en el sector bancario y por (McAlexander et al., 1994) en el sector sanitario.

La dirección de servicio incluye tres distintos aspectos: características del servicio, diseño del ambiente del servicio y entrega del servicio, en particular, las características del servicio son cualquiera de los rasgos del servicio que son ofrecidos (Neslin, 1983). El ambiente del servicio son los ajustes y propósitos requeridos para administrar el servicio (Bitner, 1992). Al contrario, la entrega del servicio es como el servicio es proporcionado en una ocasión especifica (Bitner, Booms, y Tetreault, 1990).

Estas distinciones ponen en claro que la medición de la calidad del servicio no debe solo distinguiere entre satisfacción, calidad y valor, esta debe también involucrar las características del servicio, el ambiente del servicio y la entrega del servicio (Rust y Oliver, 1994). La Figura 3 muestra el modelo de las tres componentes.

Figura 3. Modelo de los tres componentes de Rust y Oliver (1994)

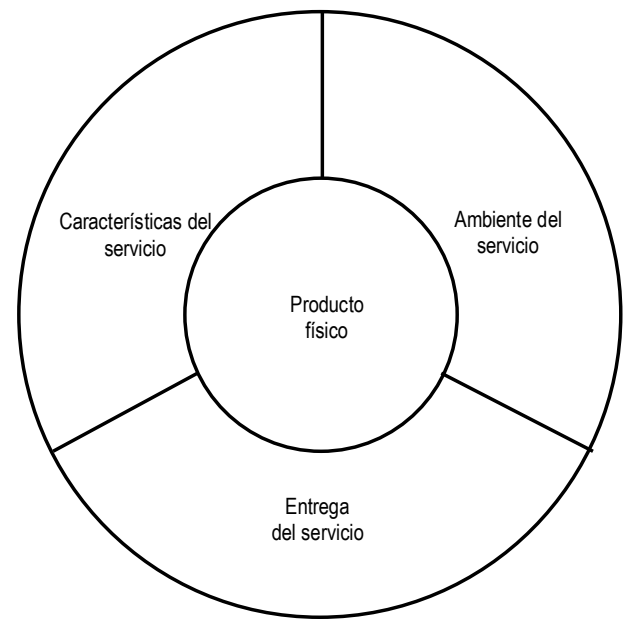




\section{Modelo jerárquico}

En el estudio de las percepciones de los usuarios de servicios, Brady y Cronin (2001) desarrollan una solución tridimensional para completar la modelización de la calidad de servicio. Entienden que no es excluyente de otros modelos pero que resulta más completo y definen la calidad de los servicios a través de tres dimensiones: la calidad de interacción, la calidad del entorno y la calidad de resultado.

- Calidad de Interacción, consiste en las actitudes de los empleados, el comportamiento entre empleado y cliente, y la formación de los profesionales que ofrecen el servicio. Ésta se refiere a la experiencia del cliente como resultado de la interacción con el elemento humano de la organización, pues la importancia de este rol de los empleados en primera línea es bien evidente en la industria de servicios (Gremler y Gwinner, 2000).

- Calidad de Entorno, se centra en el diseño de las instalaciones, las condiciones ambientales, y los factores sociales. Se refiere a los elementos físicos o tangibles de la organización y a si otros usuarios influyen en el buen desarrollo del servicio.

- Calidad de Resultado, se desarrolla en las subdimensiones de tiempo de espera, elementos tangibles y utilidad. Esta dimensión se refiere a los beneficios para el cliente o el resultado del servicio recibido, también es entendido como el producto final del servicio (Calabuig et al., 2012).

Este modelo antes mencionado se presenta en la Figura 4. 
Figura 4. Modelo jerárquico

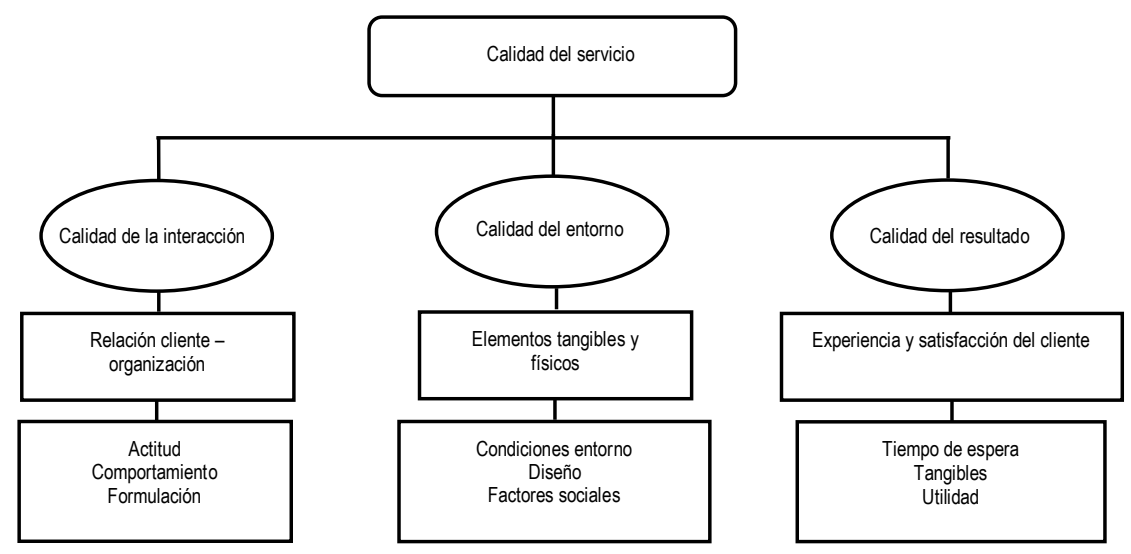

Fuente: Calabuig et al. (2012)

\section{Técnica del factor incidente critico}

Los estudios pueden ser clasificados en dos grupos, los basados en el estudio del incidente crítico (Critical Incident Factor) o los basados en la medida de los atributos de calidad de servicio (Akbaba, 2006). Los basados en la técnica del incidente crítico utilizan experiencias explicadas por los clientes sobre situaciones del servicio. Los basados en la medida de los atributos de calidad de servicio tienen como principal objetivo la medición de la calidad de servicio y los elementos que la afectan, están principalmente encabezados por el modelo SERVQUAL (Santomà y Costa, 2007).

\section{Investigaciones sobre calidad en el servicio}

A través de una revisión de investigaciones sobre calidad en el servicio se muestran las siguientes tablas que contienen los diversos estudios que se han realizado en los últimos diez años en el sector financiero, en el servicio de supermercados, entre otros sectores. 
Tabla 5. Investigaciones en el sector financiero en los últimos diez años

\begin{tabular}{|c|c|c|c|c|}
\hline Autores & $\begin{array}{c}\text { Persona } \\
\text { encuestada }\end{array}$ & Lugar & $\begin{array}{c}\text { Tamaño de } \\
\text { muestra }\end{array}$ & $\begin{array}{c}\text { Modelo } \\
\text { utilizado }\end{array}$ \\
\hline $\begin{array}{l}\text { Miguel y Flórez Romero (2007) } \\
\text { Estrategias de calidad del servicio e } \\
\text { instituciones bancarias: factores que } \\
\text { influyen en la calidad del servicio } \\
\text { percibida. }\end{array}$ & Clientes & $\begin{array}{l}\text { Cúcuta, } \\
\text { Colombia }\end{array}$ & 400 & $\begin{array}{l}\text { Escala } \\
\text { adaptada } \\
\text { al servicio } \\
\text { bancario }\end{array}$ \\
\hline $\begin{array}{l}\text { Coronado \& Parra (2007) } \\
\text { Calidad en la prestación del servicio } \\
\text { y comunicación del personal } \\
\text { bancario. Estudio empírico de } \\
\text { algunos aspectos clave para la } \\
\text { satisfacción de clientes de fondos de } \\
\text { inversión. }\end{array}$ & Clientes & $\begin{array}{l}\text { Ciudada de } \\
\text { Málago }\end{array}$ & 564 & $\begin{array}{l}\text { Escala } \\
\text { adaptada } \\
\text { al servicio } \\
\text { bancario }\end{array}$ \\
\hline $\begin{array}{l}\text { Teruel \& Garcia (2007). } \\
\text { Marketing de la prestación comercial } \\
\text { en una entidad financiera de la } \\
\text { economía social: una propuesta de } \\
\text { autoevaluación de calidad para una } \\
\text { oficina bancaria -caja de ahorro }\end{array}$ & Clientes & España & & $\begin{array}{l}\text { Escala } \\
\text { adaptada } \\
\text { al servicio } \\
\text { bancario }\end{array}$ \\
\hline $\begin{array}{l}\text { Morillo (2009). } \\
\text { La calidad en el servicio y la } \\
\text { satisfacción del usuario en } \\
\text { instituciones financieras del } \\
\text { municipio Libertador del estado } \\
\text { Mérida. }\end{array}$ & Clientes & $\begin{array}{l}\text { Libertador, } \\
\text { Merida, } \\
\text { Venezuela }\end{array}$ & 384 & servqual \\
\hline $\begin{array}{l}\text { Mejias y Manrique (2011). } \\
\text { Dimensiones de la satisfacción de } \\
\text { clientes bancarios universitarios: una } \\
\text { aproximación mediante el análisis de } \\
\text { factores. }\end{array}$ & Clientes & Venezuela & 80 & $\begin{array}{l}\text { Escala } \\
\text { adaptada } \\
\text { al servicio } \\
\text { bancario }\end{array}$ \\
\hline $\begin{array}{l}\text { González (2013). } \\
\text { Procedimientos para la evaluación de } \\
\text { la calidad percibida en los servicios } \\
\text { bancarios. }\end{array}$ & $\begin{array}{l}\text { Equipo de trabajo, } \\
\text { trabajo con } \\
\text { expertos, clientes } \\
\text { de entidades } \\
\text { bancarias }\end{array}$ & $\begin{array}{l}\text { Región } \\
\text { metopolita- } \\
\text { na de Chile }\end{array}$ & 84 & servqual \\
\hline $\begin{array}{l}\text { Arancibia, Leguina y Espinosa } \\
\text { (2013). } \\
\text { Factores determinantes en la } \\
\text { percepción de la imagen y calidad de } \\
\text { servicio y sus efectos en la } \\
\text { satisfacción del cliente. Un caso } \\
\text { aplicado a la banca chilena. }\end{array}$ & Clientes & $\begin{array}{c}\text { Región } \\
\text { metopolita- } \\
\text { na de Chile }\end{array}$ & 641 & $\begin{array}{l}\text { Escala } \\
\text { propia } \\
\text { adaptada } \\
\text { al servicio } \\
\text { bancario }\end{array}$ \\
\hline
\end{tabular}

Nota 5. Fuente: Elaboración propia 
Tabla 6. Investigaciones sobre calidad en el servicio de supermercados en los últimos diez años

\begin{tabular}{|c|c|c|c|c|}
\hline Autores & $\begin{array}{c}\text { Persona } \\
\text { encuestada }\end{array}$ & Lugar & $\begin{array}{c}\text { Tamaño de } \\
\text { muestra }\end{array}$ & $\begin{array}{c}\text { Modelo } \\
\text { utilizado }\end{array}$ \\
\hline $\begin{array}{l}\text { Pascual, Pascual, Frías y Rosel (2006). } \\
\text { Calidad de servicio en supermercados: } \\
\text { una propuesta de medición. }\end{array}$ & Clientes & $\begin{array}{l}\text { Valencia } \\
\text { España }\end{array}$ & 300 & $\begin{array}{l}\text { Escala } \\
\text { propia }\end{array}$ \\
\hline $\begin{array}{l}\text { Arroyo, Carrete y García (2008). } \\
\text { Construcción de un índice de } \\
\text { satisfacción para clientes de } \\
\text { supermercados mexiquenses Una } \\
\text { investigación exploratoria. }\end{array}$ & Clientes & $\begin{array}{l}\text { Toluca y } \\
\text { Metepec. } \\
\text { Edo. de } \\
\text { México }\end{array}$ & 1200 & $\begin{array}{l}\text { Escala } \\
\text { propia }\end{array}$ \\
\hline $\begin{array}{l}\text { Wang, Chen y Chu (2009). } \\
\text { A Study of Customer Loyalty } \\
\text { Management in Chinese Retail } \\
\text { Supermarket. }\end{array}$ & Clientes & China & 150 & $\begin{array}{l}\text { Servqual, } \\
\text { escala } \\
\text { adaptada }\end{array}$ \\
\hline $\begin{array}{l}\text { Carrete y Rosas (2010). } \\
\text { Propuesta preliminar de una escala de } \\
\text { satisfacción para clientes mexicanos de } \\
\text { supermercado. }\end{array}$ & Clientes & México & 771 & $\begin{array}{l}\text { Escala } \\
\text { propia }\end{array}$ \\
\hline $\begin{array}{l}\text { Beneke, Hayworth, Hobson y Mia } \\
\text { (2012). } \\
\text { Examining the effect of retail service } \\
\text { quality dimensions on customer } \\
\text { satisfaction and loyalty: The case of the } \\
\text { supermarket shopper. }\end{array}$ & Clientes & Sudáfrica & 307 & Servqual \\
\hline $\begin{array}{l}\text { Pedraza, Lavín, Bernal y Castillo } \\
\text { (2014). } \\
\text { Percepción de la calidad del servicio y } \\
\text { comportamientos de compra en } \\
\text { consumidores de empresas } \\
\text { comerciales. }\end{array}$ & Clientes & $\begin{array}{l}\text { Cd. Victoria } \\
\text { Tamaulipas } \\
\text { México }\end{array}$ & 200 & $\begin{array}{l}\text { Escala } \\
\text { propia }\end{array}$ \\
\hline
\end{tabular}

Nota 6. Fuente: Elaboración propia 
Tabla 7. Investigaciones sobre calidad en el servicio en otros sectores en los últimos diez años

\begin{tabular}{l} 
Estudio \\
\hline Mejías (2005). \\
Modelo para medir la calidad \\
del servicio en los estudios \\
universitarios de posgrado.
\end{tabular}

Botero y Peña (2006).

Calidad en el servicio: EI

cliente incógnito.

\begin{tabular}{ccccc} 
Sector & $\begin{array}{c}\text { Persona } \\
\text { encuestada }\end{array}$ & Lugar & $\begin{array}{c}\text { Tamaño de } \\
\text { muestra }\end{array}$ & $\begin{array}{c}\text { Modelo } \\
\text { utilizado }\end{array}$ \\
\hline Educación & Estudiantes & Venezuela & 105 & Servqual \\
$\begin{array}{c}\text { Telecomu- } \\
\text { nicaciones }\end{array}$ & $\begin{array}{c}\text { Centros de } \\
\text { atención a } \\
\text { clientes. Servicio } \\
\text { de telefonía }\end{array}$ & $\begin{array}{c}\text { Barranquilla } \\
\text { Colombia }\end{array}$ & 5 & $\begin{array}{c}\text { Formato } \\
\text { Evaluación } \\
\text { cliente } \\
\text { incógnito } \\
\text { diseñado por } \\
\text { (Botero, 2005) }\end{array}$ \\
& Clientes & Libertador, & 75 & Servqual \\
Hotelero & Merida, & & \\
& & Venezuela & & \\
Deportes & Eventos & Valencia & 2111 & $\begin{array}{c}\text { EVENTQUAL } \\
\text { (Escala propia } \\
\text { basada en }\end{array}$ \\
& España & & Método Delphi)
\end{tabular}

Morillo (2007).

Análisis de la calidad del servicio hotelero mediante la

Visión gerencial, 269-297.

Calabuig, Mundina y Crespo (2010).

Eventqual: una medida de la calidad percibida por los espectadores de eventos deportivos.

Hernández (2013).

Calidad en la prestación de servicios de salud:

Correia y Miranda (2012). DUAQUAL: calidad percibida por docentes y alumnos en la gestión universitaria.

Balmori y Flores (2014).

MECSI: Modelo para evaluar la calidad del servicio interno (MECSI: Evaluation model for internal service quality). escala del SERVQUAL.

\section{Vargas, Valecillos y}

Parámetros de medición.

Salud Usuario-paciente Ciudad de

Maracaibo

Servqual

$\begin{array}{ccccc}\text { Educación } & \begin{array}{c}\text { Docentes y } \\ \text { discentes }\end{array} & \begin{array}{c}\text { Región centro } \\ \text { de Portugal }\end{array} & 545 & \begin{array}{c}\text { DUAQUAL } \\ \text { (Adaptación de } \\ \text { servqual al } \\ \text { sector } \\ \text { universitario) }\end{array} \\ \begin{array}{c}\text { Bienes de } \\ \text { consumo }\end{array} & \text { Empleados } & \begin{array}{c}\text { Nuevo León, } \\ \text { México }\end{array} & 53 & \begin{array}{c}\text { Modelo de } \\ \text { Evaluación de la } \\ \text { Calidad al } \\ \text { servicio Interno } \\ \text { (MECSI) }\end{array} \\ & & & \\ & & \end{array}$

Nota 7. Fuente: Elaboración propia 


\section{Conclusiones}

El concepto calidad a sido utilizado por diferentes autores que le han dado diferentes definiciones, la calidad en el servicio demuestra ser un concepto subjetivo del cual se tienen diferentes acepciones y por lo tanto también se le han dado diferentes enfoques.

Se han elaborado diversos modelos de medición de calidad en el servicio cada uno de ellos con sus propias dimensiones aunque similares entre sí, pretendiendo que estas dimensiones comprendan todos los aspectos del servicio.

Los modelos de medición de calidad en el servicio se han convertido en una estrategia que permite a las organizaciones definir los aspectos del servicio que ayudaran a construir estrategias encaminadas a prestar un servicio satisfactorio, rentable y competitivo.

Prueba de que los modelos de medición se han convertido en una estrategia para las organizaciones, son todas las investigaciones que se han desarrollado en el sector servicio, por ejemplo, en el servicio financiero, el servicio de supermercados, educación, sector hotelero, salud entre otros.

La innovación en los servicios es la manera de mantenerse competitivos y de entrar o ampliar mercados; el proceso innovador en la empresas es alimentado por el conocimiento, más que por la disponibilidad de tecnología o de grandes recursos financieros; este conocimiento que sirva comercialmente y para crear valor, el cual proviene principalmente del mercado, de los competidores, de los avances tecnológicos y principalmente de los clientes (Cruz, 2008).

\section{Referencias}

Akbaba, A. (2006). Measuring service quality in the hotel industry: a study in a business hotel in Turkey, International Journal of Hospitality Management, 25, 170-192.

Arancibia, C. S., Leguina, A. \& Espinosa, Z. P. (2013). Factores determinantes en la percepción de la imagen y calidad de servicio y sus efectos en la satisfacción del cliente. Un caso aplicado a la banca chilena, Revista de Ciencias Sociales, 19(2), 255267. 
Arroyo, P., Carrete, L. \& García, S. I. (2008). Construcción de un índice de satisfacción para clientes de supermercados mexiquenses: Una investigación exploratoria, Contaduría y Administración, 225, 59-78.

Balmori, G. \& Flores, J. B. (2014). MECSI: Modelo para evaluar la calidad del servicio interno, Innovaciones en Negocios, 11(2),191-213.

Beneke, J., Hayworth, C., Hobson, R. \& Mia, Z. (2012). Examining the effect of retail service quality dimensions on customer satisfaction and loyalty: The case of the supermarket shopper, Acta Commercii, 12(1), 27-43.

Bitner, M. J. (1992): Servicescapes: The impact of physical surroundings on customers and employees, Journal of Marketing, 56, 57-71.

Bitner, M. J., Booms, B. H. \& Tetreault M. S. (1990). The service encounter: Diagnosing favorable and unfavorable incidents, Journal of Marketing, 54, 71-84

Botero, M. M. \& Peña, P. (2008). Calidad en el servicio: el cliente incógnito, Suma Psicológica, 13(2), 217-228.

Brady, M. \& Cronin, J. (2001). Some new thoughts on conceptualizing perceived services quality: a hierarchical approach, Journal of Marketing, 65(3), 34-49.

Bueno, E., Salmador, M. P. \& Merino, C. (2008). Génesis, concepto y desarrollo del capital intelectual en la economía del conocimiento: Una reflexión sobre el modelo intellectus y sus aplicaciones, Estudios de Economía Aplicada, (26)2, 43-63.

Calabuig, F., Molina, N. \& Núñez, J. (2012). Una aplicación inicial del modelo tridimensional de calidad de servicio en centros deportivos privados, E-balonmano. Journal Sport of Science, 8, 67-81.

Calabuig, F., Mundina, J. \& Crespo, J. (2010). Eventqual: una medida de la calidad percibida por los espectadores de eventos deportivos, Retos, 18, 66-70.

Camisón, C. (1999). Hacia modelos de calidad de servicio orientados al cliente en las universidades públicas: el caso de la Universitat Jaume I, Investigaciones Europeas de Dirección y Economía de la Empresa, 5(2), 69-92.

Camisón, C., Cruz, S. \& González, T. (2006). Gestión de la calidad: conceptos, enfoques, modelos y sistemas. Madrid: Pearson Educación.

Carrete, L. \& Rosas, J. A. (2010). Propuesta preliminar de una escala de satisfacción para clientes mexicanos de supermercado, Pecvnia: Revista de la Facultad de Ciencias Económicas y Empresariales, Universidad de León, (monográfico), 5-23.

CIC. (2003). Modelo intellectus: Medición y gestión del capital intelectual. [Documento 5]. Madrid: Centro de Investigación sobre la Sociedad del Conocimiento (CIC), Universidad Autónoma de Madrid.

Colmenares, O. A. \& Saavedra, J. L. (2007). Aproximación teórica de los modelos conceptuales de la calidad del servicio, Técnica administrativa, 6(32), 138-175.

Coronado, M. I. \& Parra, G. F. (2007). Calidad en la prestación del servicio y comunicación del personal bancario. Estudio empírico de algunos aspectos clave para la satisfacción de clientes de fondos de inversión. En J. C. Ayala Calvo (Ed.), Conocimiento, innovación y emprendedores: camino al futuro, España: Universidad de La Rioja, 17181732. 
Correia, S. M. \& Miranda, F. J. (2012). DUAQUAL: calidad percibida por docentes y alumnos en la gestión universitaria, Cuadernos de Gestión, 12(1), 107-122.

Cronin, J. \& Taylor, S. (1992). Measuring service quality: a reexamination an extension, Journal of Marketing, 56, 55-68.

Crosby, P. B. (1988). La organización permanece exitosa. Cd. México: Editorial McGraw-Hill.

Cruz, R. (2008). Innovación en servicios en la era del conocimiento= Services innovation in the era of knowledge, Innovaciones de negocios, 5(1), 39-52.

Cruz, J. (2004). Cuánto cuesta la calidad, Innovaciones de Negocios, 2(2), 259-276.

Dabholkar, P. A., Thorpe, D. I. \& Rentz, J. O. (1996). A Measure of Service Quality for Retail Stores: Scale Development and Validation, Journal of the Academy of Marketing Science, 24(1), 3-16.

Deming, W. E., \& Edwards, D. W. (1982). Quality, productivity, and competitive position (Vol. 183). Cambridge, MA: Massachusetts Institute of Technology, Center for advanced engineering study

Deming, W. E., \& Medina, J. N. (1989). Calidad, productividad y competitividad: la salida de la crisis. Madrid: Ediciones Díaz de Santos.

Duque, E. J. (2005). Revisión del concepto de calidad del servicio y sus modelos de medición. Innovar, Revista de ciencias administrativas y sociales, 15(25), 64-80.

Eigler, P. \& Langeard E. (1989). Servucción, el marketing de servicios. Cd. México: MacGraw-Hill.

Evans, J. (1992), A report of the total quality leadership steering committee and working councils. Milwaukee: Procter \& Gamble.

Feigenbaum, A. V. (1951), Quality control: principles, practice and administration. Nueva York: McGraw-Hill.

González, A. R. (2013). Procedimientos para la evaluación de la calidad percibida en los servicios bancarios, Ingeniería Industrial, 12(2), 43-62.

Gremler, D. \& Gwinner, K. (2000). Costumer-employee rapport in service relationships, Journal of Service Research, 3(1), 82-104.

Grönroos, C. (1984). A service quality model and its marketing implications, European Journal of Marketing, 18(4), 36-44.

Grönroos, C. (1994). Marketing y gestión de servicios: la gestión de los momentos de la verdad y la competencia en los servicios. Madrid: Editorial Díaz de Santos.

Hakansson, H. \& Snehota, I. (1989) No business is an island: The network concept of business strategy, Scandoinavian Journal of Management, 187-200.

Hakansson, H. \& Snehota, I. (2006). No business is an island, 17 years after. Scandoinavian Journal of Management, 22(3), 271-274.

Hernández, C. F. \& Ulibiarri, B. H. (2015). Calidad en el servicio y competitividad en tiendas de abarrotes, Inventio, la génesis de la cultura universitaria en Morelos, 10(22), 1-10.

Ishikawa, K. (1981), Nihonteki hinshitsu kanri. Tokio: JUSE Press Ltd. Edición inglesa: What is Total Quality Control? The Japanese way. Englewood Cliffs: Prentice-Hall, 1985. Edición española: ¿Qué es el control total de calidad? La modalidad japonesa. Bogotá: Norma, 1986. 
Jain, S. K. \& Gupta, G. (2004). Measuring service quality: SERVQUAL vs. SERVPERF scales, Vikalpa, 29(2), 25-37.

Juran, J. M. (1990). Juran y la planificación para la calidad. Madrid: Ediciones Díaz de Santos.

Kotler, P. (1997). Mercadotecnia. Cd. México: Prentice-hall.

Lloréns, F. J. (1995). Un análisis de la importancia relativa que tienen las dimensiones de la calidad de servicio en la percepción del cliente, Cuadernos de Ciencias Económicas y Empresariales, 29, 35-45.

McAlexander, J. H., Kaldenberg, D. O. \& Koenig, H. F. (1994). Service quality measurement, Journal of Health Care Marketing, 14(3), 34-40.

McDougall, G. H. \& Levesque, T. J. (1994). A Revised View of Service Quality Dimensions: An empirical Investigation. Journal of Professional Service Marketing, 11(1), 189-209.

Mejías, A. A. (2005). Modelo para medir la calidad del servicio en los estudios universitarios de postgrado, Universidad, Ciencia y Tecnología, 9(34), 81-85.

Mejías, A. A. \& Manrique, C. S. (2011). Dimensiones de la satisfacción de clientes bancarios universitarios: una aproximación mediante el análisis de factores, Ingeniería Industrial, 32(1), 1-5.

Miguel, J. A. \& Flórez, M. L. (2007). Estrategias de calidad del servicio e instituciones bancarias: factores que influyen en la calidad del servicio percibida. En Ayala, J. C. (ed.), Conocimiento, innovación y emprendedores: camino al futuro, La Rioja: Universidad de La Rioja.

Moreno, F. C., García, N. M. \& Pomar, J. N. (2012). Una aplicación inicial del modelo tridimensional de calidad de servicio en centros deportivos privados. E-balonmano. com: Revista de Ciencias del Deporte, 8(1), 67-81.

Moreno, F. C., Gómez, J. M. \& Hervàs, J. C. (2010). Eventqual: una medida de la calidad percibida por los espectadores de eventos deportivos, Retos. Nuevas tendencias en Educación Física, Deporte y Recreación, 18, 66-70.

Morillo, M. C. (2007). Análisis de la calidad del servicio hotelero mediante la escala del SERVQUAL, Visión gerencial, 6(2), 269-297.

Morillo, M. C. (2009). La calidad en el servicio y la satisfacción del usuario en instituciones financieras del municipio Libertador del estado Mérida, Economía, 34(27), 199-223.

Neslin, S. A. (1983). Designing new outpatient health services: Linking service features to subjective consumer perceptions, Journal of Health Care Marketing, 3(3), 8-21.

Nyeck, S., Morales, M., Ladhari, R. \& Pons, F. (2002). 10 years of service quality measurement: reviewing the use of the SERVQUAL instrument, The bi-annual academic publication of Universidad ESAN, 7(13), 101-107.

Oliver, R. (1980). A cognitive model of the antecedents and consequences of satisfaction decision, Journal of Marketing Research, 17, 460-469.

Oliver, R. (1981). Measurement and evaluation of the satisfaction process in retail settings, Journal of Retailing, 57(3), 25-48.

Parasuraman, A., Berry, L. \& Zeithaml, V. (1988). SERVQUAL: a multiple-item scale for measuring consumer perceptions of services quality, Journal of Retailing, 64(1), 12-40. 
Parasuraman, A., Zeithaml, V. \& Berry, L. (1985). A conceptual model of service quality and its implications for future research, Journal of Retailing, 49, 44-60.

Pascual, M., Pascual, J., Frías, M. D. \& Rosel, J. (2006). Calidad de servicio en supermercados: una propuesta de medición, Psicothema, 18(3), 661-667.

Pedraza, N. A., Lavín, J., Bernal, I. \& Castillo, L. (2014). Percepción de la calidad del servicio y comportamientos de compra en consumidores de empresas comerciales, Enfoques del estudio de calidad en el servicio, 159-174.

Philip, G. \& Hazlett, S. A. (1997). The measurement of service quality: a new P-C-P attributes model, International Journal of Quality \& Reliability Management, 14(3), pp. 260-286.

Renko, M. \& Tikkannen, J. (2002). Strategic orientations in networked hight technology product development. (Rep. Num.: 12). Findland: Turku School of Economics and Business Administrations.

Rust, R. T., \& Oliver, R. L. (1994). Service quality: New directions in theory and practice. Thousand Oaks: Sage Publications, Inc.

Santomà, R. \& Costa, G. (2011). Calidad de servicio en la industria hotelera: revisión de la literatura, Revista de Análisis turístico, 3, 27-44.

Schiffman, L. G. \& Kanuk, L. L. (2010). Comportamiento del consumidor. Cd. México: Pearson Educación.

Setó, P. D. (2004). De la calidad de servicio a la fidelidad del cliente. Madrid: ESIC Editorial.

Shewhart, W.A. (1931), Economic control of quality of manufactured product. Nueva York: Macmillan. Reeditado por la American Society for Quality Control, 1980.

Taguchi, G. (1986), Introduction to quality engineering: Designing quality into products and processes. Nueva York: Asian Productivity Organization.

Teas, R. K. (1993). Expectations, performance, evaluation and consumers perceptions of quality, Journal of Marketing, 57, 18-34.

Teruel, S. M., \& García, J. C. (2007). Marketing de la prestación comercial en una entidad financiera de la economía social: una propuesta de autoevaluación de calidad para una oficina bancaria -caja de ahorro-. En AEDEM, Decisiones basadas en el conocimiento y en el papel social de la empresa, XX congreso anual de AEDEM, Vigo: Asociación Española de Dirección y Economía de la Empresa (AEDEM), 784-796.

Vargas, V., Valecillos, J. \& Hernández, J. (2013). Calidad en la prestación de servicios de salud: Parámetros de medición, Revista de Ciencias Sociales, 19(4), 663-671.

Wang, W. C., Chen, Y. C. \& Chu, Y. C. (2009). A study of customer loyalty management in Chinese retail supermarket, International Journal of Business and Management, 4(11), 85-95.

Zailani, S. \& Rajagopal, P. (2005) Supply chain integration and performance: US versus East Asian companies, Supply Chain Management: An International Journal, 10(5), 379393. 